\title{
A backtracking evolutionary algorithm for power systems
}

\author{
Ji-Pyng Chiou ${ }^{1, a}$, Chung-Fu Chang ${ }^{2}$ and Chin-Ju Li ${ }^{1}$ \\ ${ }^{1}$ Department of Electrical Engineering, Ming Chi University of Technology, New Taipei City 24301, Taiwan \\ ${ }^{2}$ Department of Electrical Engineering, WuFeng University, Chiayi County 62153, Taiwan
}

\begin{abstract}
This paper presents a backtracking variable scaling hybrid differential evolution, called backtracking VSHDE, for solving the optimal network reconfiguration problems for power loss reduction in distribution systems. The concepts of the backtracking, variable scaling factor, migrating, accelerated, and boundary control mechanism are embedded in the original differential evolution (DE) to form the backtracking VSHDE. The concepts of the backtracking and boundary control mechanism can increase the population diversity. And, according to the convergence property of the population, the scaling factor is adjusted based on the $1 / 5$ success rule of the evolution strategies (ESs). A larger population size must be used in the evolutionary algorithms (EAs) to maintain the population diversity. To overcome this drawback, two operations, acceleration operation and migrating operation, are embedded into the proposed method. The feeder reconfiguration of distribution systems is modelled as an optimization problem which aims at achieving the minimum loss subject to voltage and current constraints. So, the proper system topology that reduces the power loss according to a load pattern is an important issue. Mathematically, the network reconfiguration system is a nonlinear programming problem with integer variables. One three-feeder network reconfiguration system from the literature is researched by the proposed backtracking VSHDE method and simulated annealing (SA). Numerical results show that the perfrmance of the proposed method outperformed the SA method.
\end{abstract}

\section{Introduction}

Recently, many mathematic algorithms have used to solve various industrial systems. Among mathematical algorithms, the evolutionary algorithms (EAs) have been widespread interest based on advantages including ease to use, robust, and so on. EAs are a class of stochastic search and optimization methods that include genetic algorithms (GAs), evolutionary programming (EP), evolution strategies (ESs), genetic programming (GP), and their variants [1]. These algorithms, based on the principles of natural biological evolution, have received considerable and increasing interest over the past decade. EAs operate on a population of potential solutions, applying the principle of survival of the fittest to produce successively better approximations to a solution. EAs are robust and suitable for effectively obtaining optima, and with a smaller probability than other algorithms of falling into local optima. The methodology of the EAs has been discussed in many literatures [2-9], and EAs are also successfully used in various industrial applications [10-15].

\footnotetext{
${ }^{a}$ Corresponding author : jipyng@ mail.mcut.edu.tw
} 
Variant methods of EAs were proposed to increase the convergence speed and the capability of global search during development of EAs. Hybrid differential evolution (HDE) [16-17] is a EAs' variant method that is one of the most excellent EAs. HDE is a stochastic search and optimization method. The fittest of an offspring competes one-to-one with that of the corresponding parent, which is different from the other evolutionary algorithms (EAs). This one-to-one competition gives rise to a faster convergence rate. However, this faster convergence also leads to a higher probability of obtaining a local optimum because the diversity of the population descends faster during the solution process. To overcome this drawback, migrating operation and accelerated operation act as a trade-off operator for the diversity of population and convergence property in HDE. Migrating operation maintain the diversity of population, which guarantees a high probability of obtaining the global optimum. And, accelerated operation is used to accelerate convergence. However, a fixed scaling factor is used in HDE. Using a smaller scaling factor, HDE becomes increasingly robust. However, much computational time should be expanded to evaluate the objective function. HDE with a larger scaling factor should results generally fall into local solution or misconvergence. Lin et al. [16] used a random number that its value is between zero and one as a scaling factor. However, a random scaling factor could not guarantee the fast convergence. The selection of mutation operator is also a very important issue in HDE. The proper mutation operator can accelerate to search out the global solution [14]. However, the selection of mutation operator is problem-dependence. In HDE, the proper mutation operator is not easy to select. To overcome the drawback of the fixed and random scaling factor and alleviate the problem of selection of mutation operator in HDE, the concept of variable scaling factor is used in variable scaling hybrid differential evolution (VSHDE) method [18-19]. The rule of updating scaling factor based on the $1 / 5$ success rule of the evolution strategies (ESs) [1,20] is used to adjust the scaling factor. The $1 / 5$ success rule emerged as a conclusion of the process of optimizing convergence rate of two functions (the so-called corridor mode and sphere model [1, 2021]). To increase the population diversity, the concept of backtracking is used in the VSHDE method (called backtracking VSHDE method).

Power systems across the world have been recently deregulated, which resulted in competition among the power sellers. This resulted in increased complexity of distribution networks. Further the distribution system planner and operator are facing new challenges that put emphasis on optimal planning and operation of the distribution system. Reduction of power loss, improvement of power quality, cost minimization and load balance among the branches are important factors for better operation of the distribution system. These can be accomplished by optimal network reconfiguration [22]. Distribution systems consist of groups of interconnected radial circuits. The configuration may be varied via switching operations to transfer loads among the feeders. Two types of switches are used in primary distribution systems. There are normally closed switches (sectionalizing switches) and normally open switches (tie switches). Those two types of switches are designed for both protection and configuration management. Network reconfiguration is the process of changing the topology of distribution systems by altering the open/closed status of switches. Because there are many candidateswitching combinations in the distribution system, network reconfiguration is a complicated combinatorial, non-differentiable constrained optimization process aimed at finding optimal operation of the distribution system. Optimal feeder reconfiguration has been investigated over decades with heuristic and meta-heuristic techniques for single and multi-objectives in order to find optimal planning and operation of the distribution system [22-28]. A multi-objective invasive weed optimization algorithm is proposed by Sudha et al. [22] to solve the optimal network reconfiguration. While solving optimal network reconfiguration of the radial distribution system, minimization of active power loss, maximum node voltage deviation, number of switching operations and the load balancing index are considered as the objectives simultaneously. Huang et al. [23] proposed parallel undirected spanning tree-based genetic algorithm (PSTGA) to solve the network reconfiguration in parallel. Liu et al. [24] proposed an improved type of immune algorithm to ensure that the algorithm can quickly converge to the global optimal solution to improve the efficiency of the algorithm solving and solution accuracy. Nguyen et al. [25] proposed a reconfiguration methodology based on a cuckoo search algorithm (CSA) for minimizing active power loss and the maximizing voltage magnitude. 
Syahputra et al. [26] proposed particle swarm optimization (PSO) algorithm based multi-objective optimization for reconfiguration of radial distribution network with the presence of distributed energy resources (DER). The benefits of DER integration in distribution system are reducing power losses, improving voltage profiles and load factors, eliminating system upgrades, and reducing environmental impacts. Sureshkumar et al. [27] presented the use of differential evolution method to reduce the losses and balancing the loads in the radial distribution network. Iswarya et al. [28] deals with reconfiguration of distribution system to minimize the power loss and considering reliability.

In this study, a backtracking VSHDE for solving the network reconfiguration of distribution systems is proposed. Here, 1/5 success rule of evolution strategies (ESs) [20-21] is used to adjust the scaling factor to accelerate searching out the global solution. In addition, the concept of backtracking used in the proposed method to increase the population diversity. One three-feeder distribution system from the literature is solved by the proposed method is sloved respectively by the proposed method and SA. From the computational results, it is observed that the convergence property of the bracktracking VSHDE method is better than that of the SA method.

\section{Backtracking VSHDE algorithm}

The backtracking VSHDE method is briefly described in the following.

Step 1. Initialization

The initial population is chosen randomly and would attempt to cover the entire parameter space uniformly as (1).

$$
\mathbf{Z}_{i}^{0}=\mathbf{Z}_{i, \text { min }}+\sigma_{i} \cdot\left(\mathbf{Z}_{i, \text { max }}-\mathbf{Z}_{i, \text { min }}\right), i=1, \ldots, N_{p}
$$

Where $\sigma_{i} \in(0,1]$ is a random number, $\mathbf{Z}_{i \min }$ and $\mathbf{Z}_{i \max }$ are the lower bounds and upper bounds of $i$ th individual, respectively. The initial process can produce $N_{p}$ individuals of $\mathbf{Z}_{i}^{0}$ randomly.

In addition, the initial historical population is also generated using equation (1) to maintain the population diversity.

Step 2. Backtracking Operation [29]

Increasing the population diversity to increase the probability of searching out the global solution is the aim of the backtracking operation. Backtracking operation has two strategies. First, two random numbers between 0 and 1 are generated. Second, if the first random number is less than the second random number, then current population is replaced with the historical population and re-permuting the every individual's position randomly in the population. Otherwise, if the first random number is greater or equal than the second random number, then current population is only re-permuting the individual's position in the historical population.

Step 3. Mutation operation

The essential ingredient in the mutation operation is the difference vector. Each individual pair in a population at the G-th generation defines a difference vector $\mathbf{D}_{j k}$ as

$$
\mathbf{D}_{j k}=\mathbf{Z}_{j}^{G}-\mathbf{Z}_{k}^{G}
$$

The mutation process at the G-th generation begins by randomly selecting either two or four population individuals $\mathbf{Z}_{j}^{G}, \mathbf{Z}_{k}^{G}, \mathbf{Z}_{l}^{G}$, and $\mathbf{Z}_{m}^{G}$ for any $j, k, l$, and $m$. These four individuals are then combined to form a difference vector $\mathbf{D}_{j k l m}$ as 


$$
\mathbf{D}_{j k l m}=\left(\mathbf{Z}_{j}^{G}-\mathbf{Z}_{k}^{G}\right)+\left(\mathbf{Z}_{l}^{G}-\mathbf{Z}_{m}^{G}\right)
$$

A mutant vector is then generated based on the present individual in the mutation process by

$$
\hat{\mathbf{Z}}_{i}^{G+1}=\mathbf{Z}_{p}^{G}+F \times \mathbf{D}_{j k l m}, i=1,2, \cdots, N_{p}
$$

Where $F$ is the scaling factor. Furthermore, $j, k, l$, and $m$ are randomly selected.

The concept of variable scaling factor used in the proposed method is to overcome the drawback of the fixed and random scaling factor. The rule of updating a scaling factor based on the $1 / 5$ success rule of the ESs is used to adjust the scaling factor. The 1/5 success rule emerged as a conclusion of the process of optimizing convergence rate of two function(the so-called corridor mode and sphere model) $[1,20-21]$.The scaling factor should be updated in every $q$ iterations as follow:

$$
F^{t+1}= \begin{cases}c_{d} \times F^{t} & \text { if } p_{s}^{t}<\frac{1}{5} \\ c_{j} \times F^{t} & \text { if } p_{s}^{t}>\frac{1}{5} \\ F^{t} & \text { if } p_{s}^{t}=\frac{1}{5}\end{cases}
$$

Where $p_{s}^{t}$ is the frequency of successful mutations measured. The initial value of the scaling factor, $F$ is set to 2 [30-31]. The factors of $c_{d}=0.82$ and $c_{j}=1 / 0.82$ [1] are used for adjustment, which should be taken place for every $q$ iterations. When the migrating operation performed or the scaling factor is too small to find the better solution, the scaling factor is reset as follow:

$$
F=1-\frac{\text { iter }}{\text { itermax }}
$$

Where iter and itermax are the number of current iteration and the maximum iteration, respectively.

Step 4. Crossover operation

The perturbed individual of $\hat{\mathbf{Z}}_{i}^{G+1}$ and the present individual of $\mathbf{Z}_{i}^{G}$ are chosen by a binomial distribution to progress the crossover operation to generate the offspring. Each gene of i-th individual is reproduced from the mutant vectors $\hat{\mathbf{Z}}_{i}^{G+1}=\left[\hat{\mathbf{Z}}_{1 i}^{G+1}, \hat{\mathbf{Z}}_{2 i}^{G+1}, \cdots, \hat{\mathbf{Z}}_{n i}^{G+1}\right]$ and the present individual $\mathbf{Z}_{i}^{G}=\left\lfloor\mathbf{Z}_{1 i}^{G}, \mathbf{Z}_{2 i}^{G}, \cdots, \mathbf{Z}_{n i}^{G}\right\rfloor$. That

$$
\hat{Z}_{g i}^{G+1}= \begin{cases}Z_{g i}^{G} & \text { if a random number }>C_{r} \\ \hat{Z}_{g i}^{G+1} & \text { otherwise }\end{cases}
$$

Where $i=1, \cdots, N_{p} ; g=1, \cdots, n$; and the crossover factor $C_{r} \in[0,1]$ is assigned by the user.

Step 5. Boundary Control Mechanism

When the gene of the child is less than the lower bound, two random numbers between 0 and 1 are generated. If the first random number is less than the second random number, the gene of child set to lower bound, otherwise, the gene of child is chosen randomly in an attempt to cover the entire parameter space uniformly. Similarly, when the gene of the child is greater than the upper bound, two 
random numbers between 0 and 1 are generated. If the first random number is less than the second random number, the gene of child set to upper bound, otherwise, the gene of child is chosen randomly in an attempt to cover the entire parameter space uniformly.

Step 6. Estimation and selection

The evaluation function of a child is one-to-one competed to that of its parent. This competition means that the parent is replaced by its child if the fitness of the child is better than that of its parent. On the other hand, the parent is retained in the next generation if the fitness of the child is worse than that of its parent, i.e.

$$
\begin{gathered}
\mathbf{Z}_{i}^{G+1}=\arg \min \left\{f\left(\mathbf{Z}_{i}^{G}\right), f\left(\hat{\mathbf{Z}}_{i}^{G+1}\right)\right\} \\
\mathbf{Z}_{b}^{G+1}=\arg \min \left\{f\left(\mathbf{Z}_{i}^{G+1}\right)\right\}
\end{gathered}
$$

Where arg min means the argument of the minimum.

\section{Step 7. Migrating operation if necessary}

In order to effectively enhance the investigation of the search space and reduce the choice pressure of a small population, a migration operation is introduced to regenerate a new diverse population of individuals. The new population is yielded based on the best individual $\mathbf{Z}_{b}^{G+1}$. The g-th gene of the i-th individual is as follows:

$$
Z_{i g}^{G+1}= \begin{cases}Z_{b g}^{G+1}+\sigma_{i} \times\left(Z_{g \text { min }}-Z_{b g}^{G+1}\right) & \text { if } \delta<\frac{Z_{b g}^{G+1}-Z_{g \text { min }}}{Z_{g \text { max }}-Z_{g \text { min }}} \\ Z_{b g}^{G+1}+\sigma_{i} \times\left(Z_{g \text { min }}-Z_{b g}^{G+1}\right) & \text { otherwise }\end{cases}
$$

Where $\sigma_{i}$ and $\delta$ are randomly generated numbers uniformly distributed in the range of [0,1]; $i=1, \cdots, N_{p} ; g=1, \cdots, n$.

The migrating operation is executed only if a measure fails to match the desired tolerance of population diversity. The measure is defined as follows:

$$
\varepsilon=\sum_{i=1, i \neq b}^{N_{p}} \sum_{g=1}^{n} \frac{\eta_{Z}}{n \times\left(N_{p}-1\right)}<\varepsilon_{1}
$$

Where

$$
\eta_{Z}= \begin{cases}0 & \text { if } \varepsilon_{2}<\left|\frac{Z_{g i}^{G+1}-Z_{b i}^{G+1}}{Z_{b i}^{G+1}}\right| \\ 1 & \text { otherwise }\end{cases}
$$

Parameter $\varepsilon_{1}, \varepsilon_{2} \in[0,1]$ express the desired tolerance for the population diversity and the gene diversity with respect to the best individual.

Step 8. Accelerated operation if necessary

When the best individual in the present generation cannot be improved any longer by the mutation and crossover operations, a decent method is then employed to push the present best individual towards attaining a better point. The accelerated phase is expressed as follows: 


$$
\mathbf{Z}_{b}^{G+1}= \begin{cases}\mathbf{Z}_{b}^{G+1} & \text { if } f\left(\mathbf{Z}_{b}^{G+1}\right)<f\left(\mathbf{Z}_{b}^{G}\right) \\ \mathbf{Z}_{b}^{G+1}-\alpha \nabla f & \text { otherwise }\end{cases}
$$

Where $\mathbf{Z}_{b}^{G}$ denotes the best individual, as obtained from equation (9). The step size $\alpha \in(0,1]$ in (13) is determined by the descent property. Initially, $\alpha$ is set to one to obtain the new individual.

Step 9. Repeat step 2 to step 8 until the maximum iteration quantity or the desired fitness is accomplished.

The main calculation procedure of the backtracking VSHDE method is as shown in Fig. 1.

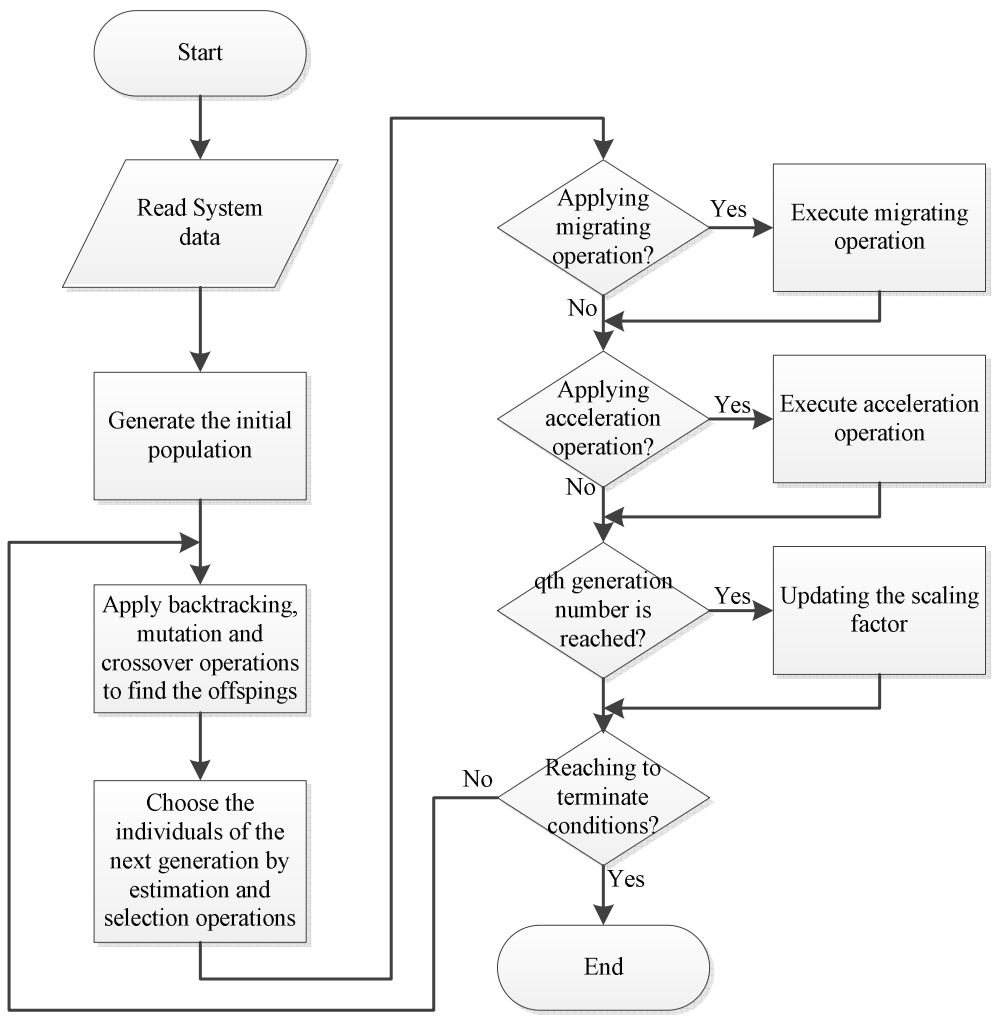

Figure 1. Main calculation procedures of the backtracking VSHDE method

\section{Problem formulation}

This network reconfiguration problem aims to minimize the system power loss, subject to operating constraints under a certain load pattern. The mathematical model of the problem can be expressed as follows:

$$
\min f=\min \left(P_{T, \text { Loss }}\right)
$$

Where $P_{T, \text { Loss }}$ is the total real power loss of the system.

The voltage magnitude at each bus must maintain within its limits. The current on each branch has to lie within its capacity rating. These constraints are expressed as follows 


$$
\begin{gathered}
V_{\min } \leq\left|V_{i}\right| \leq V_{\max } \\
\left|I_{i}\right| \leq I_{i, \max }
\end{gathered}
$$

Where

$\left|V_{i}\right|$ voltage magnitude of bus $i$

$V_{\min }, V_{\max }$ bus minimum and maximum voltage limits

$\left|I_{i}\right|, I_{i, \max }$ current magnitude and maximum current

limit of branch $i$

A set of simplified feeder-line flow formulations is employed. Considering the single-line diagram depicted in Fig.2, the following set of recursive equations is used for power flow computation [32-33].

$$
\begin{gathered}
P_{i+1}=P_{i}-P_{L i+1}-R_{i, i+1} \cdot\left(P_{i}^{2}+Q_{i}^{2}\right) /\left|V_{i}\right|^{2} \\
Q_{i+1}=Q_{i}-Q_{L i+1}-X_{i, i+1} \cdot\left(P_{i}^{2}+Q_{i}^{2}\right) /\left|V_{i}\right|^{2} \\
\left|V_{i+1}\right|^{2}=\left|V_{i}\right|^{2}-2\left(R_{i, i+1} \cdot P_{i}+X_{i, i+1} \cdot Q_{i}\right)+\left(R_{i, i+1}^{2}+X_{i, i+1}^{2}\right) \frac{\left(P_{i}^{2}+Q_{i}^{2}\right)}{\left|V_{i}\right|^{2}}
\end{gathered}
$$

Where $P_{i}$ and $Q_{i}$ are the real and reactive powers flowing out of bus $i$, and $P_{L i}$ and $Q_{L i}$ are the real and reactive load powers at bus $i$. The resistance and reactance of the line section between buses $i$ and $i+1$ are denoted by $R_{i, i+1}$ and $X_{i, i+1}$, respectively. The power loss of the line section connecting between buses $i$ and $i+1$ may be computed as

$$
P_{\text {Loss }}(i, i+1)=R_{i, i+1} \cdot \frac{P_{i}^{2}+Q_{i}^{2}}{\left|V_{i}\right|^{2}}
$$

The total power loss of the feeder, $P_{F, \text { Loss }}$, may then be determined by summing up the losses of the all line sections of the feeder. Which is given by

$$
P_{F, \text { Loss }}=\sum_{i=0}^{n-1} P_{\text {Loss }}(i, i+1)
$$

and the total system power loss, $P_{T, \text { Loss }}$, is the sum of power losses of all feeders in the system.

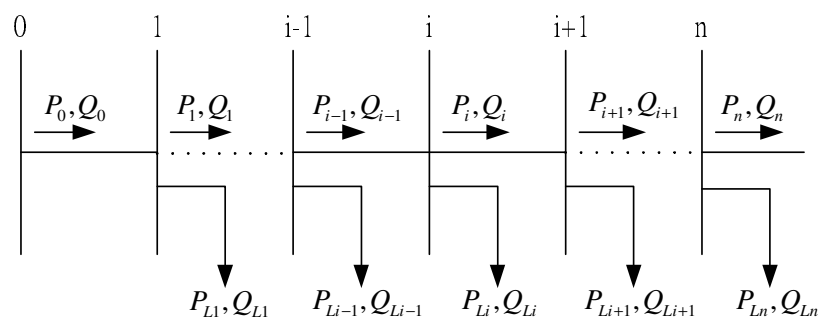

Figure 2. Single-line diagram of a main feeder. 


\section{Application of the proposed method}

Implementation of the problem begins from the parameter encoding. A tie switch and some sectionalizing switches with the feeders form a loop. A certain switch of each loop is then selected to open to make the loop become radial and the selected switch naturally become a tie switch. The network reconfiguration problem is identical to the problem of selection of an appropriate tie switch for each loop so that the power loss can be minimized. A coding scheme that recognizes the tie switch positions is proposed. The total number of tie switches is kept constant regardless of the change in the system's topology or the tie switches' positions. Fig. 3 shows an individual composing of wherein the tie switch (TS) positions. Different switches from a loop are respectively selected to cut as a tie switch to decide its associated fitness value to determine a feasible solution (radial configuration) with minimum loss. The fitness function to be maximized is defined as follows

$$
\max f=\min P_{T, \text { Loss }}=\min \sum_{k=1}^{M}\left(\sum_{i=0}^{n_{k}-1} P_{\text {Loss }}(i, i+1)\right)
$$

Where $M$ is the total feeder number of the system and $n_{k}$ is the total section number of feeder $k$.

\begin{tabular}{|l|l|l|l|}
\hline TS No.(1) & TS No.(2) & $\cdot \cdots \cdot$ & TS No.(n) \\
\hline
\end{tabular}

Figure 3. An individual composing of $n$ tie switches.

One three-feeder distribution system from the literature is investigated and the results are used to compare the performance of the proposed backtracking VSHDE method with the SA method. The Fortran SA [34] algorithm solver accessed from http://www-aig.jpl.nasa.gov/public/home/decoste /HTMLS/NN/glopt/glopt.html\#sa_codes, is used to solve the network reconfiguration of distribution systems. The SA solver recommended some setting factors for a user. The recommended factors are used for solving network reconfiguration of distribution systems. For comparison, SA package is rewritten by Matlab software.

\section{Example:}

The three-feeder distribution system [35] as shown in Fig. 4 Input data of this example system are shown in Table 1 . The system consists of 3 feeders, 13 normally closed switches, and 3 normally open switches. The system load is assumed to be constant and $S_{\text {base }}=100 M V A$. The setting factors used in backtracking VSHDE to solve this example are as follows. The population size, $N_{p}$, is set to 5 . The maximum generation, itermax, is set to 50 . The crossover factor, $C_{R}$, is set to 0.5 . Two tolerances, $\varepsilon$ and $\varepsilon_{1}$, used in the migrating operation are both set to 0.1 . The scaling factor is updating in every 10 iterations. Six strategies of mutation operation are respectively used to solve this example. To verify the performance of the proposed algorithm, this example was repeatedly solved one hundred times. Table 2 shows the best computational results. The best solutions obtained by the proposed method are same as that obtained from [36]. Number of the successful runs to achieve the best solution as [36] is $63,64,61,79,62$ and 72 for six different strategies of mutation operation used in backtracking VSHDE, respectively. From the computational results, the selection of the mutation operator in backtracking VSHDE does not affect the performance of the proposed method significantly. The SA is also applied to solve this problem. The number of times to which the best solution obtained by the SA is 35 . From the above discussion, it could be concluded that the performance of the proposed backtracking VSHDE method is better than the SA method. 


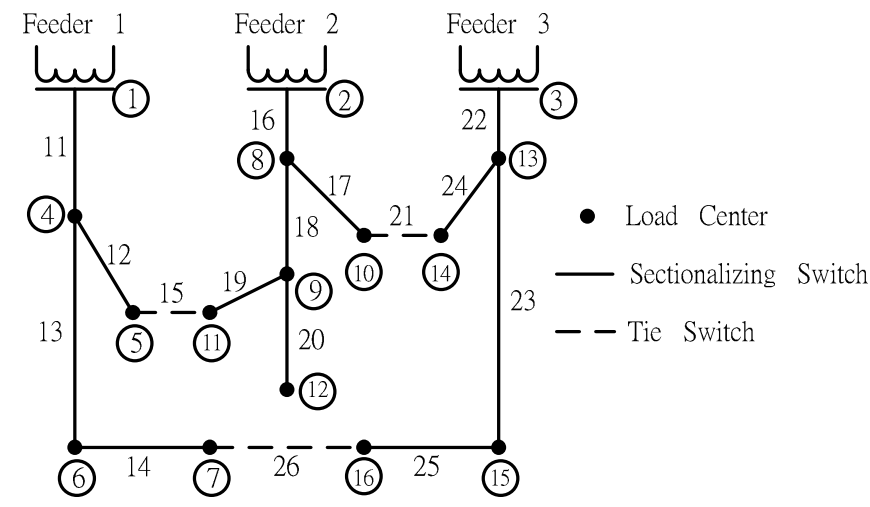

Figure 4. A three-feeder distribution system for example.

Table 1. Input data for example.

\begin{tabular}{|c|c|c|c|c|c|}
\hline Bus to bus & $\begin{array}{c}\text { Section } \\
\text { resistance }(P . U .)\end{array}$ & $\begin{array}{c}\text { Section } \\
\text { reactance }(P . U .)\end{array}$ & $\begin{array}{c}\text { End bus real } \\
\text { load }(M W)\end{array}$ & $\begin{array}{c}\text { End bus } \\
\text { reactive } \\
\text { load }(M V A R)\end{array}$ & $\begin{array}{c}\text { End bus fixed } \\
\text { capacitor }(M V A R)\end{array}$ \\
\hline $1-4$ & 0.075 & 0.1 & 2.0 & 1.6 & 1.1 \\
\hline $4-5$ & 0.08 & 0.11 & 3.0 & 1.5 & 1.2 \\
\hline $4-6$ & 0.09 & 0.18 & 2.0 & 0.8 & \\
\hline $6-7$ & 0.04 & 0.04 & 1.5 & 1.2 & 1.2 \\
\hline $2-8$ & 0.11 & 0.11 & 4.0 & 2.7 & 0.6 \\
\hline $8-9$ & 0.08 & 0.11 & 5.0 & 3.0 & 3.7 \\
\hline $8-10$ & 0.11 & 0.11 & 1.0 & 0.9 & 1.8 \\
\hline $9-11$ & 0.11 & 0.11 & 0.6 & 0.1 & \\
\hline $9-12$ & 0.08 & 0.11 & 4.5 & 2.0 & 1.8 \\
\hline $3-13$ & 0.11 & 0.11 & 1.0 & 0.9 & \\
\hline $13-14$ & 0.09 & 0.12 & 1.0 & 0.7 & \\
\hline $13-15$ & 0.08 & 0.11 & 1.0 & 0.9 & \\
\hline $15-16$ & 0.04 & 0.04 & 2.1 & 1.0 & \\
\hline $5-11$ & 0.04 & 0.04 & & & \\
\hline $10-14$ & 0.04 & 0.04 & & & \\
\hline $7-16$ & 0.09 & 0.12 & & & \\
\hline
\end{tabular}

Table 2. Numerical results of example.

\begin{tabular}{|c|c|c|}
\hline Main items & Original configuration & After reconfiguration \\
\hline Tie switches & $15,21,26$ & $19,17,26$ \\
\hline Power loss $(\mathrm{KW})$ & 511.4 & 466.1 \\
\hline \multirow{2}{*}{ Voltage Magnitude (P.U.) } & $V_{\max }=1.0000$ (Bus 1, 2,3) & $V_{\max }=1.0000$ (Bus 1, 2, 3) \\
\cline { 2 - 3 } & $V_{\min }=0.9693$ (Bus 12) & $V_{\min }=0.9716$ (Bus 12) \\
\hline Number of tie switches changed & \multicolumn{2}{|c|}{$8.86 \%$} \\
\hline Loss reduction & \multicolumn{2}{|c|}{2} \\
\hline
\end{tabular}

\section{Conclusion}

Two heuristic methods including backtracking VSHDE and SA for solving the network reconfiguration of distribution systems have been described in this work. The bactracking VSHDE 
method utilized the 1/5 success rule of the evolution strategies (ESs) to adjust the scaling factor to accelerate searching out the global solution. The variable scaling factor is used to overcome the drawback of fixed and random scaling factor used in HDE. And, the concept of backtracking is used in this paper to increasing the population diversity to increase the possibility of searching out the global solution. The computational results obtained of sloving one three-feeder distribution system from the literature is investigated. From the computational results of solving one three-feeder distribution system from the literature showed the performance of the backtracking VSHDE method is better than those obtained by the simulated annealing (SA).

\section{Acknowledgment}

Financial research support from the Ministry of Science and Technology of the R. O. C. under grant MOST 104-2221-E-131-017 is greatly appreciated.

\section{References}

1. Z. Michalewicz, Genetic Algorithms + Data Structures $=$ Evolution Programs $3^{\text {rd }}$ ed (New York, 1999)

2. I. Zelinka, A survey on evolutionary algorithms dynamics and its complexity - Mutual relations, past, present and future, Swarm and Evolutionary Computation, 25 (1), 2-14 (2015)

3. B. Kazimipour and X. Li, A.K. Qin, A review of population initialization techniques for evolutionary algorithms, Proceedings of IEEE Congress on Evolutionary Computation, 2585$2592(2014)$

4. M. Crepinsek, S.H. Liu, and M. Mernik, Exploration and exploitation in evolutionary algorithms: A survey, ACM Computing Surveys, 45 (3), 35 (2013)

5. J. Prakash, P.K. Singh, Partitional algorithms for hard clustering using evolutionary and swarm intelligence methods: a survey, Advances in Intelligent Systems and Computing, 2, 515-528 (2013)

6. B. Li, J. Li, K. Tang, and X. Yao, Many-objective evolutionary algorithms: a survey, ACM Computing Surveys, 48 (1), A10 (2015)

7. H. Ishibuchi, H. Masuda, Y. Tanigaki, and Y. Nojima, Review of coevolutionary developments of evolutionary multi-objective and many-objective algorithms and test problems, IEEE Symposium on Computational Intelligence in Multicriteria Decision-Making (MCDM), 178-184 (2014)

8. V.L. Vachhani, V.K. Dabhi, and H.B. Prajapati, Survey of multi objective evolutionary algorithms, IEEE International Conference on Circuit, Power and Computing Technologies (2015)

9. C. Von Lücken, B. Barán, and C. Brizuela, A survey on multi-objective evolutionary algorithms for many-objective problems, Computational Optimization and Applications, 58 (3), 707-756 (2014)

10. R.V. Devi, S.S. Sathya, and M.S. Coumar, Evolutionary algorithms for de novo drug design-a survey, Applied Soft Computing Journal, 27, 543-552 (2015)

11. P.M. Pradhan and G. Panda, Comparative performance analysis of evolutionary algorithm based parameter optimization in cognitive radio engine: a survey, Ad Hoc Networks, 17, 129-146 (2014)

12. M. Gen and L. Lin, Multiobjective evolutionary algorithm for manufacturing scheduling problems: State-of-the-art survey, Journal of Intelligent Manufacturing, 25 (5), 849-866 (2014)

13. S. Li, L. Kang, and X.M. Zhao, A survey on evolutionary algorithm based hybrid intelligence in bioinformatics, Bio Med Research International, 2014 (2014)

14. D.G.N. Rani and S. Rajaram, A survey on B*-Tree-based evolutionary algorithms for VLSI floorplanning optimisation, International Journal of Computer Applications in Technology, 48 (4), 281-287 (2013)

15. A. Ponsich, A.L. Jaimes, and C.A.C. Coello, A survey on multiobjective evolutionary algorithms for the solution of the portfolio optimization problem and other finance and economics applications, IEEE Transactions on Evolutionary Computation, 17 (3), 321-344 (2013) 
16. Y.C. Lin, K.S. Hwang, and F.S. Wang, Plant scheduling and planning using mixed-integer hybrid differential evolution with multiplier updating, Proceedings of the IEEE Conference on Evolutionary Computation, 1, 593-600 (2000)

17. J.P. Chiou and F.S. Wang, Hybrid method of evolutionary algorithms for static and dynamic optimization problems with application to fed-batch fermentation process, Computers \& Chemical Engineering, 23, 1277-1291 (1999)

18. J.P. Chiou, C.F. Chang, and C.T. Su, Variable scaling hybrid differential evolution for solving network reconfiguration of distribution systems, IEEE Transactions on Power Systems, 20 (2), 668-674 (2005)

19. J.P. Chiou, Variable scaling hybrid differential evolution for large-scale economic dispatch problems, Electric Power Systems Research, 77, 212-218 (2007)

20. T. Back, F. Hoffmeister, and H.P. Schwefel, A survey of evolution strategies, Proc. of $4^{\text {th }}$ Int. Conf. Genetic Algorithms, 2-9 (1991)

21. T. Back and H.P. Schwefel, An overview of evolutionary algorithms for parameter optimization, Evol. Comput., 1, 1-23 (1993)

22. D. Sudha Rani, N. Subrahmanyam, and M. Sydulu, Multi-objective invasive weed optimizationAn application to optimal network reconfiguration in radial distribution systems, International Journal of Electrical Power and Energy Systems, 73, 932-942 (2015)

23. H. Huang, J. Gu, and C. Fang, Application of undirected spanning tree-based parallel genetic algorithm in distributed network reconfiguration, Dianli Xitong Zidonghua/Automation of Electric Power Systems, 39 (14), 89-96 (2015)

24. F.C. Liu, W. Xu, G. Zhang, W.Z. Wang, and Z.Y. Li, Distribution network reconfiguration based on immune clonal selection algorithm, Environment, Proc. of 3rd International Conference on Frontier of Energy and Environment Engineering, 657-660 (2015)

25. T.T. Nguyen and A.V. Truong, Distribution network reconfiguration for power loss minimization and voltage profile improvement using cuckoo search algorithm, International Journal of Electrical Power and Energy Systems, 68, 233-242 (2015)

26. R. Syahputra, I. Robandi, and M. Ashari, PSO based multi-objective optimization for reconfiguration of radial distribution network, International Journal of Applied Engineering Research, 10 (6), 14573-14586 (2015)

27. K. Sureshkumar nad P. Vijayakumar, Distribution network reconfiguration for loss minimisation using differential evolution algorithm, ARPN Journal of Engineering and Applied Sciences, 10 (7), 2861-2866 (2015)

28. R. Iswarya and R.M. Sasiraja, Network reconfiguration of distribution system in presence of harmonic load using expert system approach, International Journal of Applied Engineering Research, 10 (55), 1961-1966 (2015)

29. P. Civicioglu, Backtracking Search Optimization Algorithm for numerical optimization problems, Applied Mathematics and Computation, 219, 8121-8144 (2013)

30. R. Storn and K.V. Price, Minimizing the real functions of the ICEC ' 96 contest by differential evolution, IEEE Conference on Evolutionary Computation, 842-844 (1996)

31. K.V. Price, Differential evolution vs. functions of the $2^{\text {nd }}$ ICEC, IEEE Conference on Evolutionary Computation, 153-157 (1997)

32. C.T. Su and C.C. Tsai, A new fuzzy reasoning approach to optimum capacitor allocation for primary distribution systems, Proc. IEEE on Industrial Technology Conf., 237-241 (1996)

33. C.T. Su, C.S. Lee, and C.S. Ho, Optimal selection of capacitors in distribution systems, Proc. IEEE Power Tech. Conf., 301 (1999)

34. B. Goffe, Global optimization of statistical functions with simulated annealing, Journal of Economics, 60 (12), 65-100 (1994)

35. S. Civanlar, J.J. Grainger, H. Yin, and S.S.H. Lee, Distribution feeder reconfiguration for loss reduction, IEEE Trans. Power Delivery, 3, 1217-1223 (1988)

36. H.C. Cheng and C.C. Kou, Network reconfiguration in distribution systems using simulated annealing, Electric Power System Research, 29, 227-238 (1994) 